\title{
Wo steht der deutschsprachige Nachhaltigkeitsjournalismus? Ein Überblick
}

\author{
Torsten Schäfer \& Stella Lorenz
}

Keywords: Nachhaltigkeitsjournalismus, Umweltjournalismus, Klimajournalismus, Impact Journalism, Magazinjournalismus, Erzählformen

\section{Abstract}

Die mediale Berichterstattung rund um den Themenkomplex »Nachhaltigkeit« hat sich sowohl im klassischen Zeitungsjournalismus als auch auf dem Zeitschriftenmarkt, den dieser Beitrag als Trendindikator exemplarisch in den Blick nimmt, fortentwickelt. Auch große Verlage setzen nun auf Heftneugründungen im »grünen « Printsegment. Gemeinsam mit bereits vorhandenen Titeln etablieren diese zum Teil alternative journalistische Erzählformen für Nachhaltigkeitsthemen. Dabei verschwimmt nicht selten die Grenze zwischen Literatur und Journalismus, wie anhand zweier Beispiele gezeigt wird. Zudem fasst der Beitrag Metastrukturen in Aus- und Weiterbildung sowie die Genese neuer grüner Onlinemedien und medialer Fachportale zusammen und macht Vorschläge für künftige Forschungsansätze zur Analyse des Netzwerks und seiner Einzelakteur*innen.

Torsten Schäfer \& Stella Lorenz: Wo steht der deutschsprachige Nachhaltigkeitsjournalismus? Ein Überblick. In: Nils S. Borchers, Selma Güney, Uwe Krüger und Kerem Schamberger (Hrsg.): Transformation der Medien - Medien der Transformation. Verhandlungen des Netzwerks Kritische Kommunikationswissenschaft. Frankfurt am Main: Westend 2021. DOI: https://doi.org/10.53291/JNRT8200.

Prof. Dr. Torsten Schäfer I Hochschule Darmstadt I torsten.schaefer@h-da.de

Stella Lorenz I Universität Freiburg und Hochschule Darmstadt I stella-marie @t-online.de 
Die deutschen Medien sind ergrünt. Sie berichten häufiger als früher über den Themenbereich »Nachhaltigkeit«, wie unter anderem Studien der Leuphana Universität Lüneburg gezeigt haben (Fischer und Hauke 2016). Dass der Begriff heute etwa doppelt so häufig in der Berichterstattung auftaucht wie noch vor 20 Jahren, wurde von Daniel Fischer und Franziska Hauke in einer Studie zur »Verwendung von >Nachhaltigkeit in deutschen Zeitungen « (Fischer und Hauke 2016) untersucht. Dieser Befund deckt sich mit dem (rein subjektiven) Eindruck, dass in den großen Medien seit etwa 2018 häufiger Themen wie Artenvielfalt und speziell Insekten Erwähnung finden. Auch die Berichterstattung zum Klimawandel hat - nach dem Extremsommer 2018 und den seit 2019 anhaltenden Fridays-for-Future-Protesten - deutlich zugenommen.

Ganz unabhängig davon besetzen neuere und neue Printmagazine wie enorm, transform, Futurzwei oder oya die grüne Nische, die auch durch den Erfolg der Landhefte mit ihrer idealisierten Sicht auf Naturund Umweltthemen breiter geworden ist. Schon deutlich länger gibt es Zeitschriften wie das Ökologisches Wirtschaften, die Politische Ökologie oder die Umweltbriefe, die sich als Fachmagazine in den 1980er Jahren etabliert haben und nach wie vor halten. Auch online sind zu verschiedenen Aspekten von Nachhaltigkeit in den vergangenen zehn Jahren zahlreiche neue Portale entstanden wie Klimareporter, Utopia, Karmakonsum, WiWoGreen oder Biorama sowie die klimajournalistische Nachwuchsplattform Klimareporter.in aus Österreich. ${ }^{1}$ Einige Portale wie Perspective Daily entstammen dem sogenannten »Konstruktiven Journalismus«, der bewusst Ideen, Lösungen und Erfolge transportiert - besonders gern aus dem alternativen und grünen Milieu und eben öfter mit Bezug zu Klima, Energie und Nachhaltigkeit.

Im Folgenden wird gezeigt, welchen Veränderungen der deutsche Nachhaltigkeitsjournalismus ${ }^{2}$ unterliegt - zunächst unter der Per-

1 Einen Überblick über jene Portale gibt es beispielsweise auf der Website futurphil.de (Oestreicher 2020).

2 Der in der Wissenschaft noch nicht allzu lange verwendete Begriff des Nachhaltigkeitsjournalismus wurde vorrangig von Gerd Michelsen und Daniel Fischer im Rahmen entsprechender Forschungen an der Leuphana Universität Lüneburg geprägt. Er fasst »journalistische Aktivitäten zusammen, die einen Beitrag zur Debatte des Konzepts einer nachhaltigen Entwicklung leisten beziehungsweise sich darauf beziehen« (Michelsen und Fischer 2016, 10). Synonym, 
spektive der relevanten Zeitschriftengründungen sowie darauffolgend mit der Analyse weiterer Strukturmerkmale, etwa Aus- und Weiterbildungsmöglichkeiten, Medienpreise oder Studiengänge.

\section{Aus der Praxis: Das Zeitschriftenregal als Transformationsindikator}

Seit dem ersten Erscheinen der Landlust 2005, das magazinjournalistisch gesehen als Initialzündung für einen neuen Naturdiskurs (wenn auch mit eskapistischen Tendenzen) ${ }^{3}$ gesehen werden kann, hat sich der Printmagazinmarkt in diesem Bereich stark entwickelt. Über 50 neue Publikumsmagazine ${ }^{4}$ sind an den Markt gegangen, die sich in einem ähnlichen Themengebiet bewegen (siehe Tab. 1). Ein ganz eigenes Segment haben die zahlreichen, in den letzten zehn Jahren neugeschaffenen Publikationen begründet: Unter dem von Schäfer, Kasper und Austen (2015) vorgeschlagenen Komplex »Sinn + Muße« lassen sich rund 30 Magazine mit breitgefächerten Schwerpunkten verorten: angefangen bei der Achtsamkeitspublikation Flow (und zahlreichen Nachahmern) über vegane Ernährungs- und Kochzeitschriften bis hin zu Testballons wie der autonom gegründeten und unabhängig von Werbeanzeigen finanzierten Zeitschrift transform, deren Macher*innen als Journalist*innenkollektiv arbeiten.

aber auch ergänzend dazu werden oft auch die Begriffe »Umwelt-« oder »Klimajournalismus«, »Grüner Journalismus « und »Transformationsjournalismus« verwendet (vgl. Krüger in diesem Band). Auch Impact-Journalismus (vgl. Borner, Oxenfarth und Ronzheimer 2019) weist ähnliche Charakteristika wie der Nachhaltigkeitsjournalismus auf.

3 Die Landlust und viele der anschließend veröffentlichten Landhefte zeichnen sich vor allem durch eine sehr idyllische Naturdarstellung aus. Problematische Themen wie Klimawandel oder Umweltverschmutzungen spielen keine Rolle. Die Beschreibung des Familienmagazins LandKind bringt es auf den Punkt: »Die Rückbesinnung auf die einfachen, alltäglichen Dinge des Lebens und ein Leben im Einklang mit der Natur stehen dabei immer im Vordergrund. LandKind stillt die heutige Sehnsucht nach einer heilen Welt [...].« (Panini Verlags GmbH 2019; Hervorhebungen TS \& SL).

4 Unter dem Begriff der Publikumsmagazine werden solche Hefte verstanden, deren Zielgruppe (im Gegensatz zu den oftmals sehr spezifisch ausgerichteten Fachmagazinen) eine gesamtgesellschaftliche ist und die neben der Wissensvermittlung auch der Unterhaltung und Beratung dienen (Menhard und Treede 2004, 21-22). 
Die Übersicht, die im Rahmen der laufenden Dissertation der Mitautorin dieses Beitrages entstanden ist, will einen Überblick über die Publikationen des von Schäfer, Kasper und Austen (2016) geschaffenen Segments geben, umfasst aber darüber hinaus auch die davor bereits etablierten Magazine, die sich in ihrer Grundausrichtung mit ökosozialem Wandel, der Sehnsucht nach einem alternativen Leben, Verbundenheit (also mit Achtsamkeit im weitesten Sinne) und Nachhaltigkeit beschäftigen und mindestens einen dieser Werte auch vermitteln. ${ }^{5}$

Tab. 1: Aktuell erhältliche Publikumszeitschriften mit Nachhaltigkeits- und Achtsamkeitsbezug seit 1976 (eigene Darstellung).

\begin{tabular}{|l|l|l|}
\hline Magazinname & Launch & Claim \\
\hline info3 & 1976 & Bewusst leben. Gesellschaft gestalten. \\
\hline natur & 1980 & $\begin{array}{l}\text { Das Magazin für Umwelt, Natur und ein besseres } \\
\text { Leben. }\end{array}$ \\
\hline Kraut und Rüben & 1984 & Biologisch gärtnern, natürlich leben. \\
\hline Ökotest & 1985 & - \\
\hline natur\&heilen & 1986 & Die Monatszeitschrift für gesundes Leben. \\
\hline Greenpeace Magazin & 1993 & - \\
\hline BIO & 1994 & Das Magazin von Körper, Geist und Seele. \\
\hline NaturApotheke & 1994 & Das Magazin für Gesundheit und bewusstes \\
\hline bewusster leben & Leben. \\
\hline Landlust & 2003 & Sei gut zu dir! \\
\hline natürlich & 2005 & Schönes Landleben. \\
\hline Liebes Land & 2006 & Gut für mich. \\
\hline Futurzwei (zeozwei) & 2008 & Das Beste vom Land. \\
\hline Kochen ohne Knochen & 2009 & Dagazin für Zukunft und Politik. \\
\hline
\end{tabular}

5 Reine »Glücksmagazine«, bei denen Meditation, psychologische Themen und Spiritualität (wie etwa bei happinez oder Happy Way) im Vordergrund stehen, wurden aus diesem Grund nicht mit in die Betrachtung aufgenommen, ebenso wie etwa Yoga-Hefte oder Wander-Magazine als Special-Interest-Magazine mit Bezug auf ein spezielles Hobby. 


\begin{tabular}{|c|c|c|}
\hline Magazinname & Launch & Claim \\
\hline Landleben & 2009 & Lebensstil mit Liebe zur Natur. \\
\hline Landldee & 2009 & Land erleben und genießen. \\
\hline köstlich vegetarisch & 2010 & Die neue Lust am Kochen. \\
\hline Mein schönes Land & 2010 & Gutes bewahren, Schönes entdecken. \\
\hline Land und Berge & 2010 & Die schönste Art, die Natur zu erleben. \\
\hline Servus in Stadt \& Land & 2010 & Einfach. Gut. Leben. \\
\hline Meine gute Landküche & 2010 & Ländlicher Genuss und regionale Vielfalt. \\
\hline enorm & 2010 & Die Zukunft fängt bei Dir an. \\
\hline oya & 2010 & anders denken. anders leben. \\
\hline LandGenuss & 2011 & Die besten Gerichte der Saison! \\
\hline Landldee - Wohnen und Deko & 2011 & Natürliche Wohnideen für zuhause. \\
\hline Landldee - Landapotheke & 2012 & Heilen und pflegen nach alter Tradition. \\
\hline LandKind & 2012 & Landglück für die ganze Familie. \\
\hline Landzauber & 2012 & Die Vielfalt des Landlebens. \\
\hline Flow & 2013 & $\begin{array}{l}\text { Eine Zeitschrift ohne Eile, über kleines Glück und } \\
\text { das einfach Leben. }\end{array}$ \\
\hline evolve & 2013 & Magazin für Bewusstsein und Kultur. \\
\hline veganmagazin & 2014 & - \\
\hline vegan world & 2014 & - \\
\hline Slow Food (Relaunch) & 2014 & Genießen mit Verstand. \\
\hline Mein schöner Landgarten & 2014 & Gärtnern im Einklang mit der Natur. \\
\hline Mein schönes Landhaus & 2014 & Ländlich wohnen. Natürlich leben. \\
\hline emotion slow & 2014 & Runterkommen. Genießen. Einfach leben. \\
\hline Welt Vegan Magazin & 2015 & - \\
\hline Land \& Leute Edition & 2015 & Die schönen Seiten unseres Landes. \\
\hline Vegan für mich & 2015 & Glücklich, fit und Spaß dabei. \\
\hline Walden & 2015 & Abenteuer vor der Haustür. \\
\hline
\end{tabular}




\begin{tabular}{|c|c|c|}
\hline Magazinname & Launch & Claim \\
\hline ma vie & 2015 & Die Kunst, sich Zeit zu nehmen. \\
\hline greenLIFESTYLE & 2015 & Das Magazin für einen nachhaltigen Lebensstil. \\
\hline Biouty & 2015 & Das einzigartig natürliche Beauty-Magazin. \\
\hline Werde & 2015 & Wir leben das Leben. \\
\hline transform & 2015 & Magazin für das Gute Leben. \\
\hline maas & 2016 & Impulse für ein erfülltes Leben. \\
\hline Food \& Farm & 2016 & Wissen, was man isst. \\
\hline Slowly Veggie & 2016 & Vegetarisch \& vegan genießen. \\
\hline ma vie - Gesund leben & 2016 & Die beste Medizin für mich! \\
\hline Anderswo & 2016 & Europa nachhaltig entdecken. \\
\hline greenup & 2016 & Nachhaltiger leben! \\
\hline $\begin{array}{l}\text { Mein schönes Land - Grüne } \\
\text { Apotheke }\end{array}$ & 2017 & Gesundheit aus der Natur. \\
\hline hygge & 2017 & Vom Glück, das Leben mit anderen zu teilen. \\
\hline Landldee - Altes Wissen & 2018 & Omas Tipps \& Kniffe wieder neu entdeckt. \\
\hline carpe diem & 2019 & Zeit für ein gutes Leben. \\
\hline Wohllebens Welt & 2019 & Ein neuer Blick auf die Natur. \\
\hline Brigitte Be Green & 2019 & Das Magazin für ein nachhaltiges Leben. \\
\hline Vegan Food \& Living & 2019 & Das vegane Lifestyle-Magazin. \\
\hline $33 \%$ & 2020 & Das Waldmagazin. \\
\hline Landldee - natürlich leben & 2020 & Einfach nachhaltige Tipps für jeden Tag \\
\hline
\end{tabular}

Neu ist auch, dass die Mainstream-Printmedien Nachhaltigkeit als Trendthema verstärkt aufgreifen. So erschienen 2019 mit Brigitte Be Green und Wohllebens Welt (beide aus dem Verlagshaus Gruner + Jahr) gleich zwei neue Magazine für unterschiedliche Zielgruppen im breiteren Publikum (Altrogge 2019; Matzig 2019). Das Interesse daran ist offenbar groß: Der Verlag meldete bei beiden Erstausgaben Verkaufszahlen von rund 45000 (Wohllebens Welt) beziehungsweise 50000 Exemplaren (Brigitte Be Green) (Tusch 2019; Borgböhmer 2019). 
Dass sich Printmagazine für die Vermittlung von komplexeren Fragestellungen aus dem Nachhaltigkeitskontext, sei es Energiewende, Artensterben oder Mobilität, besonders gut eignen, hängt einerseits mit der Beschaffenheit des Mediums Zeitschrift an sich zusammen: Denn sie stehen per se mit ihren langen Beiträgen für Tiefe und Detailfülle in der Darstellung, sind das Medium des Hintergrunds und kombinieren dies zudem noch mit einer ausgeprägten Visualität, was das Verständnis komplexer Stoffe erleichtert. Sie müssen ob der analogen Form, die nicht selten auch besonders ästhetisch, haptisch und optisch ansprechend daherkommt, mit Zeit und Muße konsumiert werden; entsprechend gut stehen die Chancen, dass durch die intensive Rezeption Inhalte nachhaltig im Gedächtnis bleiben. Andererseits bieten Zeitschriften auch journalistischen Spielraum: Sie sind durch ihre thematische Ausrichtung sowie den gratwandernden Charakter zwischen unterhaltendem und wissensvermittelndem Medium freier in der Themenpräsentation, in Erzählformen und vor allem auch in der Gestaltung als beispielsweise Tageszeitungen. Zeitschriften sollen »ihre Leser emotional erreichen, sie begeistern «, hebt der Wissenschaftsjournalist Martin Meister im Interview mit Torsten Schäfer $(2012,57)$ hervor. Die Basis, um transformativen Inhalten die notwendigen »Strukturen, Erfahrungs- und Resonanzräume (Borner et al. 2019, 19) einzurichten, welche die Voraussetzung für nachhaltiges Handeln ist, scheint bei Magazinen schon von Grund auf gegeben.

Vor allem im Bereich des Nachaltigkeitsjournalismus ist eine zwar grundsätzlich sachlich-kritische, aber dennoch engagierte journalistische Haltung oder vielleicht sogar Meinung zum Themenkomplex präsenter als in anderen Bereichen und weicht von der Neutralität ab, die etwa im Nachrichtenjournalismus den journalistischen Berufsethos auszumachen scheint. ${ }^{6}$ Vielmehr ist Neutralität gegenüber den Themen Umweltschutz und Nachhaltigkeit kontraproduktiv für ein Magazin, das sich vorrangig mit diesen Themen beschäftigt: Nicht wenige der Magazingründer*innen - insbesondere die der unabhängigen, »nischigen« Hefte wie oya, transform oder Futurzwei - haben ihre Publikationen aus einer Überzeugung heraus gegründet und machen diese auch

6 Diesen Zwiespalt haben beispielsweise auch Humburg, Fischer, Marwege und Michelsen in ihrer Delphi-Studie (2013) untersucht. Die befragten Journalist*innen sahen darin einerseits die Notwendigkeit, ihr eigenes Engagement sichtbarer zu machen, um auch die Leser*innen zu aktivieren, waren sich aber des »Rollenkonflikts« (ebd., 23) bewusst. 
in ihren journalistischen Ausdrucksformen deutlich, ohne dabei pathetisch, mahnend oder lediglich unterhaltend zu wirken.

Das Problem des Klimawandels, dessen Lösung ein achtsameres Handeln der Gesellschaft voraussetzt, muss so kommuniziert werden, dass es auch für fachfremde Leser*innen verständlich ist. Die wissenschaftliche Komplexität von Klima-, Umwelt- und Nachhaltigkeitsthemen so zu vermitteln, dass möglichst viele Rezipierende sich für sie interessieren, im besten Fall selbst aktiv werden und so mitunter die für eine Nachhaltigkeitswende unabdingbaren politisch-strukturelle Reformen mit anstoßen, erfordert neue Journalismusformen. ${ }^{7}$ In diesem Zusammenhang taucht im Kontext der aktuellen Debatte zu Nachhaltigkeit und Journalismus in einer Sondierungsstudie des Umweltbundesamtes (Borner et al. 2019) der passende Begriff dazu auf, der zuvor im deutschsprachigen Raum kaum präsent war: ${ }^{8}$ »Impact-Journalismus « - ein Journalismus, der Leser*innen nicht nur zu einer Meinung, sondern auch zu einem Handlungswillen verhilft. Bezeichnend für ihn sind qualitativ hochwertige journalistische Arbeit, saubere Recherche und gleichzeitig eine Nähe zu den Rezipierenden, die letztere einbindet und sie den Journalismus mitgestalten lässt (ebd., 26-28). Aber auch literarische Formen kommen im Magazinjournalismus verstärkt zum Tragen - nicht zuletzt in Form von Nature Writing, das seinen Ursprung im englischsprachigen Raum hat und insbesondere in der hiesigen Literaturszene stark an Bedeutung gewinnt (vgl. Schäfer 2018). ${ }^{9}$

Ansätze des daraus resultierenden transformativen Charakters werden in vielen der neueren Magazine nicht nur inhaltlich, sondern auch

7 Damit ist selbstredend nicht nur die persönliche Verhaltensveränderung im Alltag gemeint, sondern auch die Anregung zu politischem Engagement. Dass grundlegende, wortwörtlich nachhaltige, gesamtgesellschaftliche Veränderungen geschehen und sich festgefahrene wirtschaftliche Strukturen auflösen, ist im Wesentlichen Aufgabe der Politik. Dafür ein Bewusstsein herzustellen muss ebenso Ziel des Nachhaltigkeitsjournalismus sein.

8 Das französische Start-up Sparknews etablierte den Begriff 2013 im Zusammenhang mit handlungsaktivierenden Geschichten, als es erstmals einen »Impact Journalism Day« veranstaltete (vgl. Krüger in diesem Band).

9 Henry David Thoreaus Selbsterfahrungsroman Walden (1854) gilt als Schlüsselwerk, das den Impuls gab, real Erlebtes in der Natur - also subjektive Erfahrungen - literarisch zu verarbeiten. Dabei werden auch naturwissenschaftliche Fakten eingeflochten. In vielerlei Hinsicht sind Ansätze von Nature Writing daher auch im Journalismus, oft in der Form der Reportage, zu finden und eignen sich gut, um ökologische Themen auf literarische Weise aufzuarbeiten. 
in der Erzählform sichtbar. Zwei Fallbeispiele sollen dies im Folgenden verdeutlichen.

\subsection{Poetische Sprache und visuelle Gestaltung bei Wohllebens Welt}

Im 2019 innerhalb der GEO-Markengruppe neu erschienenen Naturmagazin Wohllebens Welt wird im Beitrag »Weder Wasser noch Land « von Rainer Harf anschaulich gezeigt, wie literarische Charakteristika in bereits etablierte journalistische Formen (in diesem Fall ist es ein Feature mit szenischem Einstieg) eingeflochten werden können.

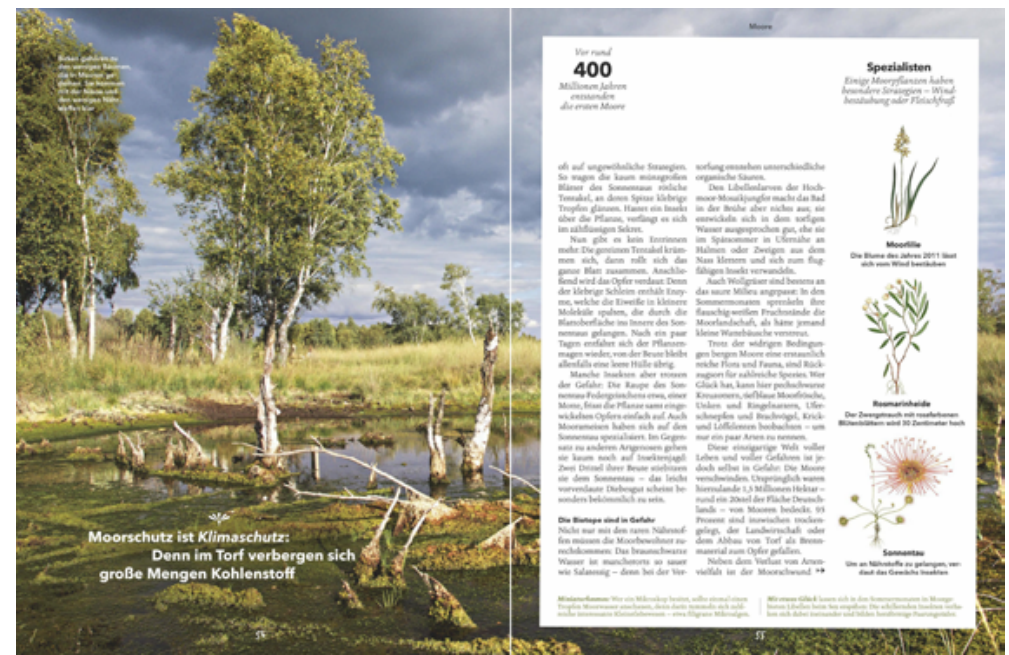

Abb. 1: Zahlreiche Formen der Wissensvermittlung finden sich auf dieser Doppelseite des Beitrags von Rainer Harf in Wohllebens Welt (Harf 2019, 54-55).

Der Artikel, in dem es um Moorlandschaften, deren Funktion im Ökosystem und deren Erhalt geht, spricht die Leser*innen zunächst visuell mit seitenfüllenden, fast schon romantischen Bildern an. Auch neben dem Fließtext ist viel auf den Seiten zu entdecken: Naturwissenschaftliche Pflanzenzeichnungen, Trivia-Beiträge zum Thema unten auf der Seite sowie hervorgehobene Zitate und Zahlen. Im Text selbst ist vor allem der erzählerische, literarisch anmutende Einstieg bemerkenswert: 
Bei jedem Schritt ertönt ein Gurgeln und Schmatzen. Nebelschwaden wabern über den morastigen Grund. Wie erstarrte Figuren ragen die Silhouetten knorriger Birken aus dem Riedgras. Hin und wieder unterbrechen seltsame Laute die gespenstische Stille: »Bu bu bu bu« - der Ruf einer Sumpfohreule. (Harf 2019, 51)

Die Poesie der Naturklänge dient hier als Vehikel für die Phantasie der Leser*innen und erleichtert es ihnen, sich auf die Thematik einzulassen. Gleichwohl ist der Beitrag reich an Fakten:

Da die abgestorbenen Pflanzen nur unvollständig verwesen und sich zu mächtigen Lagen aufschichten, entweicht der in ihnen gebundene Kohlenstoff nicht als Kohlendioxid in die Luft - so wie bei allen anderen verwesenden Pflanzen. Obwohl sie weltweit nur drei Prozent der Landfläche ausmachen, speichern Moore so viel Kohlenstoff wie alle Wälder der Erde zusammen. (Harf 2019, 56)

Die Komplexität des Verwesungsvorgangs nicht unerwähnt zu lassen ist Teil der Strategie zur Wissensvermittlung. Mit Hilfe der konsequent angewandten Erzählform versucht der Autor, die Informationsaufnahme der Leser*innen möglichst effektiv zu gestalten. Er schließt die Klammer mit einem literarischen Ausstieg: »Und dann kann man sie wieder genießen: die magische Aura dieser tückischen Lebensräume.« (Harf 2019, 56)

\subsection{Das Märchen als journalistischer Schreibmodus bei transform}

Ebenfalls erzählerisch, allerdings ohne visuelle Unterstützung und durchweg literarisch aufgezogen ist der Beitrag »\#HansImGlück « von Hans Rusinek (2018) in der werbeunabhängig und ausschließlich mit Crowdfunding produzierten Zeitschrift transform. Der Autor schildert darin konsequent im Märchenerzählstil die Geschichte von Hans im Glück in der modernen, kapitalistischen Welt und vermittelt den Leser*innen anhand dessen die Folgen der Konsumsucht sowie die Möglichkeit eines besitzlosen und dennoch freudvollen Lebens. Dabei greift er gleichermaßen auf den typischen Sprachduktus eines Märchens wie auf Begriffe und Phänomene des 21. Jahrhunderts zurück:

»Wohl wahr, lieber Hans, mein ist eine Bahncard 100. Auf ihrer Gunst fahre ich in allen Zügen, wann es mir beliebt. Ingleichen trenne ich mich vom 
sperrigen Besitz, mein Luxus ist die Reise.«— »Ei, was gäb ich darum, solch eine Bahncard mein eigen zu nennen!«, sagte Hans, den planetaren Grenzen seines Konsums bewusst. (Rusinek 2018, 62)

Im gleichen ungewöhnlichen Kontext werden außerdem Handlungsoptionen in Bezug auf Mobilität und Umweltauswirkung vermittelt:

»Hört, Hans«, sprach der [Sitz-]Nachbar und zeigte ihm ein Instagram-Abbild seines Rennrads, »euch zu Liebe will ich tauschen und euch das Rad für die Karte lassen. Bedenkt doch: Hier [beim Bahnfahren im ICE; TS \& SL] wird die Energie aus ungezählten Kohlekraftwerken durch die Trasse gejagt. Dekarbonisierung sieht anders aus. Das Rad schont die Umwelt und ihr reist mit eigener Kraft.« (Rusinek 2018, 63)

Die fiktionale Erzählung greift real existente Umstände auf und präsentiert sie in einer bekannten Form - die Kombination von Märchen und ökosozialer Betrachtung allerdings ist ungewöhnlich. Sie löst Neugier auf den Ausgang der Geschichte aus und regt zur Reflexion an: Wie hat der Autor die populären Eckpfeiler der Grimm'schen Erzählung auf die heutige Zeit übertragen? Was bedeutet das für mich und mein Handeln? Der Text transportiert Fakten, Unterhaltung und Handlungsoptionen gleichermaßen und stellt damit ein gelungenes Beispiel für den Ausbruch aus gewohnten journalistischen Formaten dar.

\subsection{Wie weiter? Literarisches Erzählen als Chance für Printmagazine}

Nicht nur die grüne Printmagazinlandschaft entwickelt sich stark weiter, auch der darin enthaltene Journalismus ist im Umbruch: Haltungsverdeutlichung, erzählerische Experimente und partizipative Ansätze mit Leser*innen kommen vermehrt vor und zeigen, dass und wie sich Nachhaltigkeit in ihrer ganzen Dimension in Publikumszeitschriften positioniert.

Die beiden Fallbeispiele verdeutlichen, wie verschieden die Durchschlagskraft und die Schnelligkeit dieses Wandels ablaufen kann: Während Wohllebens Welt als Publikation eines tradierten Verlagshauses noch recht behutsam auf literarische Erzählformen (in diesem Fall Nature Writing) zurückgreift, kann das unabhängige Magazin transform in Bezug auf fiktionales Erzählen aus den Vollen schöpfen (ohne den journalistischen Anspruch zu vernachlässigen). In jedem Fall zeigt 
sich, dass Magazine offen sind für Literarischen Journalismus, wie ihn Eberwein (2013, 144, 181, 188-189) beschreibt, und diesen praktisch nutzen: Kreativität, Subjektivität und die Tendenz, bestehende journalistische Konventionen aufzubrechen, sind prägende Merkmale.

Letztlich sind dies nur zwei Exempel für einen in Transformation begriffenen Nachhaltigkeitsjournalismus, der sich neben experimentelleren, literarischen Erzählformen zudem durch eine veränderte, haltungsstärkere Rolle der Schreibenden auszeichnet. Insbesondere angesichts des tendenziell steigenden Interesses der Mainstream-Medien an diesen Inhalten darf die weitere Umsetzung mit Spannung verfolgt werden. Mit Blick auf den sukzessiven gesellschaftlichen Wandel verändern sich möglicherweise die Vorzeichen für kleinere Magazine, die den Aufbruch als Chance nutzen könnten. Dass für Journalist*innen, die sich in diesem Bereich betätigen, die Voraussetzungen stimmen, ist dabei essenziell. Letztere haben sich in Teilen positiv entwickelt, wie im Folgenden anhand von neu entstandenen Metastrukturen für den praktischen Nachhaltigkeitsjournalismus gezeigt wird. Dabei geht es um Fachportale, Medienpreise, Aus- und Weiterbildungsmöglichkeiten, Berufsverbände und Hochschulangebote.

\section{Eine neue Infrastruktur aus Projekten, Plattformen, Stiftungen und Studiengängen ${ }^{10}$}

In den vergangenen zehn Jahren ist eine Infrastruktur entstanden, die das grüne Mediengeschehen bewertet, fördert und untersucht. Getragen werden die Forschungsprojekte, Rechercheplattformen, Studiengänge und Seminare von Hochschulen und Akademien, oft finanziert mit privaten Geldern und Stiftungsvermögen. Ebenfalls zugenommen hat die Zahl grüner Journalist*innenpreise. Derer 13 zählt das Portal journalistenpreise.de mittlerweile, darunter auch ein eigener Umweltjournalismus-Preis für Österreich.

Eines der jüngsten Elemente dieser Infrastruktur ist das Netzwerk Weitblick (NWW), ein Verein, der sich seit März 2015 für Nachhaltigkeit im Journalismus einsetzt. Derzeit sind mehr als 60 Journalist*innen und andere Medienschaffende sowie Wissenschaftler*innen dabei, von ARD und Deutschlandfunk bis FAZ und Tagesspiegel. Sie wollen Re-

10 Dieser Abschnitt ist eine überarbeitete Version eines Beitrags auf der Website klimafakten.de (Schäfer 2019b). 
cherchehilfen, Seminare sowie Stipendien anbieten und als Ansprechpartner*innen für Kolleg*innen dienen. Das Vorstandsteam hat ein zweijähriges Qualifizierungsprojekt samt Publikationsreihe entwickelt, um insbesondere jungen Journalist*innen zugehöriges Handwerk und Wissen in verschiedenen Feldern des Nachhaltigkeitsjournalismus vermitteln zu können. An vielen Akademien und Hochschulen ist das NWW mit seiner Weiterbildungsreihe präsent und damit zum bislang wichtigsten Anbieter von nachhaltigkeitsjournalistischen Seminaren geworden. Andere Initiativen, wie etwa die Initiative Nachhaltigkeitsjournalismus in Österreich, sind kleiner und weniger sichtbar.

Übrigens haben sich im NWW erstmals überhaupt deutschsprachige Journalist*innen im Feld von Umwelt und Nachhaltigkeit zusammengetan. In anderen Ländern war dies schon viel früher der Fall, beispielsweise in den USA mit der einflussreichen Society of Environmental Journalists (SEJ), aber etwa auch in Frankreich, Brasilien, Schweden, Indonesien, Kenia und Tansania.

\subsection{Gemeinsam publizieren: Synergien online nutzen}

Während beim NWW vor allem das Vernetzen und Fördern im Mittelpunkt steht, haben sich andere Journalist*innen explizit zum gemeinsamen Publizieren zusammengeschlossen: Eines der bekanntesten Beispiele ist das genossenschaftlich organisierte Onlineportal krautreporter.de, das sich durch Leserbeiträge finanziert und seine Artikel nur für Mitglieder zugänglich macht. Ohne eine generelle Bezahlschranke publizieren hingegen riffreporter.de, eine Gemeinschaft freier Journalist*innen, die in kleinen Gruppen themenspezifisch arbeiten und jeweils ihr Sachgebiet in einer »Koralle « online bündeln, um dort ihre Texte in Teilen zum Kauf anzubieten. Unter anderem gibt es thematische Kanäle für die Berichterstattung über spezielle Bereiche wie Vögel und Flüsse ebenso wie allgemeinere, etwa über das Klima.

Sowohl die Klima-Koralle der Riffreporter als auch klimareporter.de sind interessante, neue Stränge innerhalb des neuen medialen Netzwerkes der Nachhaltigkeit. Denn dort - ganz so wie bei oya und auch der Politischen Ökologie zur Nachhaltigkeit insgesamt - sind neue Einblicke und das ganze Tableau der Themen aus Klimapolitik, -forschung und Energiewende zu bekommen. Eine bewährte Recherchequelle auf Papier ist zudem die Zeitschrift Umwelt aktuell und die dazugehörige Website eu-koordination.de des Deutschen Naturschutzrings (DNR). Beide 
Medien informieren lückenlos vor allem über die EU-Perspektive von Klima- und Umweltthemen. Diese Sichtweise ist so kompakt sonst kaum zu finden und gerät bei Recherchen häufig in Vergessenheit. Ein weiterer Kanal speziell für den europäischen Blick ist das mehrsprachige Portal Euractiv mit eigenen Rubriken für Energiepolitik und Klimawandel.

Eng verknüpft mit dem erwähnten Netzwerk Weitblick ist die Plattform grüner-journalismus.de (die Torsten Schäfer, Mitautor dieses Beitrags, leitet). Das Projekt versucht seit Anfang 2014 von der Hochschule Darmstadt aus, die Berichterstattung zu Umwelt- und Nachhaltigkeitsthemen zu fördern - mit Themendossiers, Linklisten, einem Blog oder auch Interviews mit Umweltjournalist*innen und Forscher*innen. Das Geld zum Aufbau kam von der privaten Stiftung Forum für Verantwortung des früheren Metro-Vorstandes Klaus Wiegandt. Mittlerweile muss sich die Plattform selbst tragen. Entwickelt hat die Idee der Kommunikationswissenschaftler Peter Seeger, der schon seit Jahren dem Nachwuchs Nachhaltigkeit vermittelt. Noch immer, sagt er, gebe es große Defizite, etwa bei Themenauswahl und Blickwinkel, insbesondere in den großen Massenmedien: „Thematisiere ich eine vermeintliche >Wachstumsschwäche der Wirtschaft unhinterfragt - oder gelingt es, das Konzept von Wirtschaftswachstum kritisch zu betrachten und mit der Ressourcenund Verteilungsfrage zu verknüpfen?« (Seeger, zitiert in Schäfer 2019b)

\subsection{Aus- und Weiterbildungsmöglichkeiten für Nachhaltigkeitsjournalist*innen}

Auch die Aus- und Weiterbildungslandschaft hat sich weiterentwickelt: Das Deutsche Journalistenkolleg bietet mittlerweile eine ganze Reihe zum Umweltjournalismus an. Dazu kommen Medienseminare zu Themen wie Klima, Energiewende oder Verkehr, die Stiftungen, Umweltverbände oder Beratungsagenturen anbieten. Ein Beispiel dafür ist die Sommeruniversität zu Storytelling und Klimawandel des Climate Culture Communications Lab in Brandenburg. Aber auch die RobertBosch-Stiftung bot im Rahmen ihrer Förderlinien wie etwa der Masterclass für Wissenschaftsjournalist*innen schon ganze Themenjahre zu Klima und Energie an. Fachverbände wie die Wissenschaftspressekonferenz machen ebenso klimajournalistische Weiterbildungsangebote für Medien wie Institutionen aus der Wissenschaft selbst, sei es das Potsdam-Institut für Klimafolgenforschung, der Deutsche Wetterdienst oder der Think Tank Ecologic. 
Entsprechende Fachseminare bieten nun die Hochschulen in Ansbach und Nürnberg an. Etabliert sind solche Veranstaltungen und Module schon in Darmstadt, an der FH Rhein-Sieg in St. Augustin sowie der Universität Dortmund, die seit 2013 den Medien-Doktor Umwelt betreibt. In diesem Projekt begutachten Fachjournalist*innen (zeitweise darunter auch Torsten Schäfer, Mitautor dieses Artikels) anhand von 14 Qualitätskriterien die Umweltberichterstattung deutscher Medien. Die Gelder stammen auch hier von der privaten Wilo-Foundation, hinter welcher der Dortmunder Pumpensystemhersteller Opländer steht.

Stiftungsgelder sind zunächst ein Segen, der aber meist nicht ewig währt. Sie schieben an, sichern vielleicht auch ab, garantieren aber meist keine dauerhafte Unterstützung. Eine langfristig tragfähige Mischfinanzierung scheint beispielsweise die gemeinnützige Rechercheplattform correctiv.org gefunden zu haben - angeschoben mit Geldern der Essener Brost-Stiftung, akquiriert sie inzwischen Mittel sowohl von anderen Stiftungen als auch privaten Unterstützer*innen, zu denen große Internetkonzerne wie etwa Google oder Facebook zählen.

Dauerhafter können Hochschulen und Universitäten arbeiten - aber eben vor allem in der Forschung und Ausbildung. Gerade die kommunikationswissenschaftliche Erforschung des Klimajournalismus hat sich in den vergangenen zehn Jahren stark entwickelt; zu nennen sind hier etwa die Universitäten in Hamburg, Ilmenau, Lüneburg, Dortmund und Zürich, aber auch Hochschulen in Nürnberg und Darmstadt.

Auch Universitäten ohne journalistische Bezüge haben sich in die Debatte eingebracht: Die Forschungsstelle für Umweltpolitik der FU Berlin hat unter der Leitung von Roland Zieschank zusammen mit dem umtriebigen Berliner Wissenschaftsjournalisten Manfred Ronzheimer Medienseminare zur Großen Transformation ins Leben gerufen, zu denen Aktivist*innen, Forscher*innen, Journalist*innen und Stiftungspersonal in den Jahren 2016 und 2017 erschienen und die Vernetzung des losen, grünmedialen Netzwerks weiter voranbrachten.

Überhaupt Berlin: Hier finden die meisten Seminare und Konferenzen zum Themenkreis statt, im Juli 2019 etwa ein Workshop zu Klimawandel und Sprache, den das Netzwerk Degrowth-Journalismus und die taz-Panther-Stiftung organisierten. Damit zeigten sie, welche Qualität und Tiefe die Vernetzung mittlerweile erreicht hat. Denn es ging, wie so oft in den vergangenen Jahren, nicht mehr um die grundlegende Frage, wie Medien über Klima oder Energie berichten und wie sie dies besser machen könnten. Sondern die eingeladenen Journalist*innen, darunter GEO-Reporterin Johanna Romberg und Johannes Heimrath 
von oya, diskutierten stundenlang um sperrige Begriffe und mögliche alternative Wortschöpfungen für die Klimakommunikation. Hintergrund waren neue Sprachregelugen, die etwa der britische Guardian für seine Klimaberichterstattung jüngst etabliert hatte (Carrington 2019).

Die Metadebatte zum Klimajournalismus differenziert sich also aus. Was fehlt, ist noch ein eigener Studiengang in diesem Bereich, wie es ihn an der Universität von Boulder (Colorado) in den USA gibt, aber auch im schwedischen Jonköping. Den einzigen eigenständigen Ausbildungsgang bot, oft in Wochenendkursen, bisher die Leuphana Universität Lüneburg mit dem 2012 gestarteten, berufsbegleitenden Zertifikat »Nachhaltigkeit und Journalismus « an (der Autor war daran beteiligt). Inzwischen liegt das Programm auf Eis, es fanden sich zuletzt zu wenige interessierte Berufstätige, die bereit waren, rund 4500 Euro an Gebühren zu bezahlen. Einige frühere Absolvent*innen haben zwischenzeitlich das Netzwerk Weitblick mitgegründet, insofern haben die Lüneburger Aktivitäten strukturelle Spuren hinterlassen - aber auch wissenschaftliche, denn in Lüneburg entstand unter Daniel Fischer und Gerd Michelsen ein eigener Forschungsbereich, der sich Nachhaltigkeit und Medien widmet.

\section{Zwischenfazit: Hoffnungsvolle Entwicklungen, wenige Ressourcen}

Was hat das vielfältige Netzwerk rund um den grünen Journalismus bisher erreichen können? Nützlich sind sicherlich die zahlreichen Recherchehilfen und Themendossiers auf manchen Websites. In welchem Umfang diese tatsächlich genutzt werden und welche Auswirkung sie auf den praktischen Journalismus haben, müsste näher untersucht werden. Ein Indikator für Erfolg ist die beobachtbare Vertiefung und Differenzierung von Debatten. So geht es auf Podien bisweilen speziell um Sprache im Klimajournalismus - und nicht mehr nur um Klimajournalismus im Allgemeinen. Hoffnungsvoll stimmen gerade auch die Aktivitäten von jungen Kolleg*innen wie im Fall des Netzwerk Degrowth-Journalismus. Ebenso positiv ist die Beobachtung, dass sich augenscheinlich immer mehr Bachelor- und Masterkandidat*innen für nachhaltigkeitsjournalistische Themen und Fragen der medialen Nachhaltigkeit entscheiden, was aber ebenfalls übergreifend analysiert werden müsste.

Vielleicht ist es noch zu früh, um ein Fazit zu ziehen. Der eingangs zitierte Daniel Fischer, der die Lüneburger Projekte mit aufgebaut 
hat, weist darauf hin, dass der Journalismus erst vergleichsweise spät begonnen habe, sich intensiver mit Nachhaltigkeit und deren Einzelthemen zu befassen - »viel später als etwa der Bildungssektor oder die Wissenschaft« (Fischer, zitiert in Schäfer 2019b).

Verändert hat der Medien-Doktor Umwelt bereits die Sicht auf die deutsche Umweltberichterstattung. Denn nach einer Analyse der ersten 50 Beiträge, jeweils anhand von 13 Kriterien begutachtet, wird klar, wo es klemmt und was Medien gut machen: Recht selten werden Umweltprobleme aufgebauscht oder verharmlost und es fanden sich auch nur in sechs Artikeln klare Faktenfehler wie etwa falsche Zahlen. Häufiger versäumen es Journalist*innen, verschiedene Standpunkte zu recherchieren; Tendenzen zur Einseitigkeit sind erkennbar. Oft mangelt es an kundiger Einordnung: Selbst wenn beispielsweise der $\mathrm{CO}_{2}$-Ausstoß eines Landes korrekt angegeben wird, erklärt die Zahl für sich genommen noch wenig - sie müsste in Bezug gesetzt werden zu Vorjahren, anderen Staaten oder Zielmarken (vgl. Rögener 2015).

Das größte Problem ist laut Medien-Doktor Umwelt aber fehlender Kontext: 42 von 50 Zeitungsartikeln, Radioanalysen und Onlinestücken berichteten relativ eng gefasst und ereignisfixiert über Umweltprobleme und setzten sie nicht weiter in Bezug zu wirtschaftlichen, sozialen oder politischen Hintergründen. Dahinter dürften Mängel beim Sachwissen zum Klimawandel stehen, aber etwa auch zur EU-Dimension der Umwelt- und Klimapolitik (vgl. ebd.). Ein Grund für die fatale »Dekontextualisierung «, wie Forscher*innen es nennen, ist aber auch das Mehrfachparadoxon, in dem Journalist*innen heute oft arbeiten: Sie sollen mit weniger Personal in kürzerer Zeit komplexer werdende und noch in der Zahl zunehmende Themen zu einer insgesamt besseren Qualität mit Hilfe immer neuer Technologien fortwährend attraktiver produzieren. Im Ergebnis fehlt vor allem eine Ressource: Zeit - für Recherche und Faktencheck, für Weiterbildung und Wissenserwerb. Dies gilt vor allem für kleinere und regionale Medien.

Dasselbe lässt sich für den Nachhaltigkeitsjournalismus konstatieren, zu dessen Verbesserung es unterm Strich neue Ideen der Finanzierung braucht, gerade im Hinblick auf kleine und regionale Medien, die über die Einzelthemen aus dem Nachhaltigkeitskontext berichten sollen. In Skandinavien gibt es erfolgreiche Systeme der öffentlichen Medien- und Presseförderung durch unabhängige Räte, die staatliche Gelder meist an kleinere Regionalmedien geben - um explizit die publizistische Vielfalt in der Provinz zu sichern (Schäfer 2019a). Solche Modelle könnten auch für Deutschland diskutiert werden im Hinblick auf eine Ausweitung und 
Verbesserung etwa des Klimajournalismus, der im Zuge der Klimafolgen (Waldsterben, Wassermangel, Landwirtschaft und Trockenheit) zunehmend lokaler werden wird und dem vor dem Hintergrund der fortschreitenden Klimanot eine immer größere Verantwortung zukommt.

\section{Literatur}

Altrogge, Georg. 2019. »Waldbaden« mit Wohlleben als Magazin: Warum es höchste Zeit ist, dass wir alle mal einen Baum umarmen. Meedia.de vom 18. April, https://meedia.de/2019/04/18/waldbaden-mit-wohlleben-als-magazinwarum-es-hoechste-zeit-ist-dass-wir-alle-mal-einen-baum-umarmen/. Zugegriffen: 25. August 2020.

Borgböhmer, Thomas. 2019. Mehr als 55.000 Exemplare: G+J meldet Verkaufszahlen für Premierenausgabe von »Brigitte be Green«. Meedia.de vom 10. Dezember, https://meedia.de/2019/12/10/mehr-als-55-000-exemplare-gj-meldet-verkaufszahlen-fuer-premierenausgabe-von-brigitte-be-green/. Zugegriffen: 25. August 2020.

Borner, Joachim, Anke Ochsenfarth und Manfred Ronzheimer. 2019. Impact-Journalismus und zielgenaues Storytelling für gesellschaftlichen Wandel. Sondierungsstudie des Umweltbundesamtes. Berlin: Kolleg für Management und Gestaltung nachhaltiger Entwicklung GmbH, Umweltbundesamt. http://kmgne.de/wpcontent/uploads/2019/12/Sondierungsstudie-des-Umweltbundesamtes-2019. pdf. Zugegriffen: 25. August 2020.

Carrington, Daniel. 2019. Why the Guardian changes the language it uses about the environment. The Guardian vom 17. Mai, https://www.theguardian.com/ environment/2019/may/17/why-the-guardian-is-changing-the-language-ituses-about-the-environment/. Zugegriffen: 25. August 2020.

Eberwein, Tobias. 2013. Literarischer Journalismus. Theorie - Traditionen - Gegenwart. Köln: Herbert von Halem.

Fischer, Daniel. 2020. Nachhaltigkeitskommunikation. In: Nachhaltigkeit - interdisziplinär. Konzepte, Diskurse, Praktiken. Ein Kompendium, herausgegeben von Evi Zemanek und Ursula Kluwick, 42-55. Köln: UTB Böhlau.

Fischer, Daniel, und Franziska Hauke. 2016. Die Verwendung von »Nachhaltigkeit« in deutschen Zeitungen: Ergebnisse einer empirischen Medienanalyse. In: Nachhaltigkeit und Journalismus. Erkenntnisse und Impulse aus Wissenschaft und Praxis, herausgegeben von Gerd Michelsen und Daniel Fischer, 55-70. Bad Homburg: Verlag für Akademische Schriften.

Fischer, Daniel, und Thomas Stollenwerk. 2016. Das gute N-Wort. Grüner Journalismus vom 28. Dezember, https://gruener-journalismus.de/studie-begriffnachhaltigkeit/. Zugegriffen: 27. August 2020.

Harf, Rainer. 2019. Weder Wasser noch Land. Wohllebens Welt Nr. 2, 46-56.

Matzig, Gerhard. 2019. Weltretten ist Frauensache. Süddeutsche Zeitung vom 14. 
Oktober 2019, https://www.sueddeutsche.de/medien/frauenzeitschrift-brigitte-be-green-nachhaltigkeit-1.4638443/. Zugegriffen: 25. August 2020.

Menhard, Edigna, und Tilo Treede. 2004. Die Zeitschrift. Von der Idee bis zur Vermarktung. Konstanz: UVK.

Michelsen, Gerd, und Daniel Fischer (Hrsg.). 2016. Nachhaltigkeit und Journalismus. Erkenntnisse und Impulse aus Wissenschaft und Praxis. Bad Homburg: Verlag für Akademische Schriften.

Oestreicher, Fabian (Hrsg.). 2020. Nachhaltigkeitsmedien. https://futurphil.de/ medien/nachhaltigkeitsmedien/. Zugegriffen: 30. September 2020.

Panini Verlags GmbH (Hrsg.). 2019. Mediadaten LandKind 2020, https://blaufeuer.com/wp-content/uploads/LandKind_Mediadaten_2020.pdf. Zugegriffen: 30. September 2020.

Rögener, Wiebke. 2015. Umweltjournalismus. Einbettung in den Kontext fehlt. European Journalism Observatory vom 15. Oktober 2015, https://de.ejo-online. eu/qualitaet-ethik/umweltjournalismus-einbettung-den-kontext-fehlt/. Zugegriffen: 25. August 2020.

Rusinek, Hans. 2018. \#HansImGlück. transform Nr. 5, 62-63.

Schäfer, Torsten. 2019a. Zeitungssterben. Lösungsansätze aus Skandinavien. Fachjournalist.de vom 29. August, https://www.fachjournalist.de/zeitungssterbenloesungsansaetze-aus-skandinavien/. Zugegriffen: 30. September 2020.

Schäfer, Torsten. 2019b. Wo steht der deutschsprachige Umwelt- und Klimajournalismus? Ein Überblick. klimafakten.de vom 16. September, https://www.klimafakten.de/meldung/wo-steht-der-deutschsprachige-umwelt-und-klimajournalismus-ein-ueberblick/. Zugegriffen: 27. August 2020.

Schäfer, Torsten, und Martin Meister. 2012. Es gibt eine gewisse Borniertheit. In: Umwelt Europa. Grüne Gesellschaft und europäische Krise - neue Fragen an den Journalismus, herausgegeben von Carla Schulte-Reckert und Torsten Schäfer, 52-59. Berlin: Friedrich-Ebert-Stiftung Journalistenakademie.

Schäfer, Torsten, Lena Kasper, und Felix Austen. 2015. Der Kiosk als sozialer Spiegel: Wertewandel und Zeitschriftenmarkt. Fachjournalist.de vom 23. Januar, https://www.fachjournalist.de/der-kiosk-als-sozialer-spiegel-wertewandelund-zeitschriftenmarkt/. Zugegriffen: 25. August 2020.

Schäfer, Torsten. 2018. Erfolgsfaktor Natur - Das Genre Nature Writing. Fachjournalist.de vom 13. Dezember, https://www.fachjournalist.de/erfolgsfaktor-natur-das-genre-nature-writing/. Zugegriffen: 30. September 2020.

Tusch, Robert. 2019. Gruner + Jahr: Naturmagazin »Wohllebens Welt« übertrifft zum Start die Erwartungen. Meedia.de vom 1. Juli, https://meedia. de/2019/07/01/gruner-jahr-naturmagazin-wohllebens-welt-uebertrifft-zumstart-die-erwartungen/. Zugegriffen: 25. August 2020.

\section{Open Access}

Dieser Beitrag erscheint unter der Creative-Commons-Lizenz CC BY-ND 3.0 DE: https://creativecommons.org/licenses/by-nd/3.0/de/. 